\title{
Meta-analysis of the efficacy of pulmonary surfactants combined with budesonide for the prevention of bronchopulmonary dysplasia
}

\section{Bingbin He}

Department of pediatric

Jiayong Zhuang

Department of pediatric, The sixth hospital of huizhou

Boran Ye

Department of pediatric, The sixth hospital of Huizhou

Guosheng Liu ( $\nabla$ tlgs@jnu.edu.cn )

Research article

Keywords: Pulmonary surfactant; Budesonide; Bronchopulmonary dysplasia; Meta-analysis.

Posted Date: July 29th, 2019

DOI: https://doi.org/10.21203/rs.2.12087/v1

License: (c) (i) This work is licensed under a Creative Commons Attribution 4.0 International License.

Read Full License 


\section{Abstract}

Background Pulmonary surfactants(PS) combined with the intratracheal instillation of budesonide to prevent bronchopulmonary dysplasia (BPD) have been report previously. However, the safety of the use of PS combined with budesonide is still unknown and remains to be clarified. Methods PubMed, the CochraneLibrary, EMBASE, the China Knowledge Network and the Wanfang database were searched for relevant studies. Searches were performed from December 2018, and data from randomized controlled trials (RCTs) were collected. Primary outcome measures were BPD incidence, BPD-related mortality. Secondary outcomes were BPD-related complications. The Cochrane risk assessment tool was used for the evaluation of bias. RevMan5. 3software was employed for meta-analysis. An Egger's test was used for publication bias assessments. Results A total of 720 subjects were enrolled from 6 RCTs, including 352 in the experimental group and 368 in the observation group. A meta-analysis showed that the incidence of BPD [RR=0.42, 95\% $\mathrm{Cl}(0.37,0.89), \mathrm{P}<0.001]$ and BPD-related mortality $[\mathrm{RR}=0.54,95 \% \mathrm{Cl}$ $(0.38,0.89), P<0.05]$ significantly differed between the groups. Differences were also observed for intraventricular hemorrhage, infection/sepsis, retinopathy of prematurity (ROP), and patent ductus arteriosus. There were no significant differences in the incidence of PDA, neonatal necrotizing enterocolitis (NEC), hyperglycemia, or hypertension $(P>0.05)$. Conclusion The intratracheal instillation of pulmonary surfactants with budesonide can reduce the incidence of BPD and BPD-related mortality, with no increased risk of short-term complications. However, considering both sample size and study bias, the safety and efficacy of this treatment plan requires clarification in large-samples, and multi-center clinical RCTs. In addition, the impact of long-term complications such as neurodevelopmental disorders requires further assessment.

\section{Background}

Broncho pulmonary dysplasia (BPD) occurs during the early stages of neonatal development. Due to immature lung tissue oxygen inhalation or mechanical ventilation in response to chronic lung injury, BPD impacts neonatal prognosis and pulmonary function[1-3]. The disease was first proposed by northway and colleagues in the 1960s[4]. Due to continual improvements in the diagnosis and treatment of premature infants and critical newborns in China, the survival rates of children with BPD have gradually improved. To-date, a lack of unified norms for clinical diagnosis and disease treatment exist. Clinicians can effectively treat neonatal respiratory disease that is characterized by progressive dyspnea with the prophylactic application of corticosteroids. The pharmacological basis is that glucocorticoids induce newborns to produce pulmonary surfactants, thereby reducing pulmonary resistance and increasing the concentration of other drugs in the alveoli[5]. Considering that premature infants with low birth weights account for more than $50.5 \%$ of children with BPD, methods to reduce its incidence remains an area of intense research interest[6]. Animal experiments have confirmed that the intratracheal infusion of pulmonary surfactants combined with budesonide can effectively increase the concentration of budesonide in the alveoli of experimental animals, increasing the anti-inflammatory effects of the drug[7]. However, based on relevant studies in China and other countries, newborns of small gestational age and 
low birth weight differ in terms of their response and tolerance to pulmonary surfactants when combined with budesonide.

Although prenatal glucocorticoid protocols, the postpartum application of pulmonary surfactants (PS), and protective ventilation strategies have improved survival in preterm infants, the incidence of BPD remains high. Light BPD is common in immature preterm infants of a gestational age $<28$ weeks and birth weights $<1000 \mathrm{~g}$.BPD remains a common lung disease that leads to premature death and long-term illness [8].The etiology of BPD is complex, with lung inflammation and host immune responses considered major pathogenic factors.Due to the anti-inflammatory effects of glucocorticoids, their postpartum intravenous administration in premature infants can reduce the incidence and severity of BPD [9], but this leads to complications, including gastrointestinal bleeding, intestinal perforation, and elevated blood pressure. High blood sugar levels, infections, and those with cerebral palsy are not recommended for intravenous administration [10]. As the local application of glucocorticoids in the airways directly affects bronchial tubes and lungs, it can replace intravenous infusion to reduce the incidence of BPD in preterm infants. However, safe and effective drug delivery methods require further analysis. This article evaluates the safety and efficacy of pulmonary surfactants in combination with the intratracheal instillation of budesonide to prevent BPD by meta-analysis.

\section{Methods}

\section{Literature inclusion criteria}

(1) Type of study: Randomized controlled trial (RCT); (2) Subject: Birth weight $<1500 \mathrm{~g}$ and chest radiograph diagnosed as respiratory distress syndrome (RDS); (3) Intervention: Test groups were treated with intratracheal instillation using a mixture of pulmonary surfactants and budesonide. Control groups were treated with intratracheal instillation of pulmonary surfactants.

(4) At least one outcome index set was reported; (5) The language was not limited.

\section{Exclusion criteria}

(1) Non-RCTs (reviews, animal experiments, in vitro experiments, retrospective studies, observational studies, repeated studies; (2) non-intratracheal instillation: e.g. aerosolized; (3) Research topics were irrelevant and could not be obtained in the literature.

\section{Outcome indicators}

The primary outcome index was the BPD incidence rateand BPD related mortality. The secondary outcome indicator was the BDP related complication rate, including intraventricular hemorrhage, infection/sepsis, the retinopathy of prematurity (ROP), patent ductus arteriosus (PDA), neonatal necrotizing enterocolitis(NEC), high blood sugar levels and high blood pressure.

\section{Literature Search Strategy}


Articles were retrieved from PubMed, the Cochrane Library, EMBASE, the China Knowledge Network and Wanfang Medical Databases. The search time was from creating database to July 2019. We collected data on the use of pulmonary surfactants combined with budesonide intratracheal instillation to prevent bronchopulmonary dysplasia. Relevant literature on safety and efficacy were compiled. Search terms included bronchopulmonary dysplasia, chronic lung disease, chronic pulmonary disease, BPD, CLD, and lung dysplasia. Chinese search terms included bronchopulmonary dysplasia, pulmonary surfactants, glucocorticoids, budesonide, and primec.

\section{Document screening, data extraction and quality evaluation}

The literature was screened by two independent researchers. Data extraction, literature quality evaluations, and cross-checking were performed. If the opinions were not uniform, disagreements were resolved by discussions. Duplicated literature were removed and titles and abstracts were excluded if not related to the study subject, or included non-RCTs. Full texts were screened to determine inclusion.

Data extraction included: (1) first author and year of publication; (2) year of inclusion, inclusion criteria, BPD diagnostic criteria and general information (gestational age, birth body) quality, gender, mode of production, prenatal use of hormones for pregnancy; (3) intervention methods such as time of administration and dose; (4) Outcome indicators.

Quality evaluation of the clinical trials was performed using the Cochrane risk bias assessment tool. Assessments were based on the following: (1) whether random sequences were generated and are correct; (2) whether to distribute concealment; (3) whether to study blindness of the subjects, and whether the outcome was influenced by the lack of blinding; (4) whether to blind the study results; (5) Whether the outcome measures were complete; (6) whether selective reports, loss of follow-up, or shedding cases are described.

\section{Statistical methods}

Meta-analyses were performed using RevMan5. 3 software. Heterogeneity tests were used for Q assessments and 12 statistics. If $P \geq 0.1,12 \leq 50 \%$, no heterogeneity existed between the studies, and the combination was a fixed effect; if $\mathrm{P}<0.1,12>50 \%$, heterogeneitywas present and combined with a random effects model. Count data were expressed as the relative risk ratio (RR) and $95 \%$ confidence intervals $(\mathrm{Cl})$. For the two categorical variables, the odds ratio $(\mathrm{OR})$ and its $95 \% \mathrm{Cl}$ were used to evaluate the safety and efficacy of the intervention. Publication bias assessments were performed using Egger's tests. $P<0.05$ was considered statistically significant.

\section{Results}

\section{Literature Search}

A total of 512 articles were retrieved, including 43 articles from the China Knowledge Network, 35 from the Wanfang Medical Network, 112 from PubMed, 254 from EMBASE, and 68 from the Cochrane Library. 
Six screens were included. The screening process is shown in Figure 1.

\section{Basic information for study inclusion}

All 6 RCTs were intratracheal instilled with PS and budesonide as the experimental group. Only PS was administered into the tracheas of the control group. A total of 720 subjects were included; 352 in the experimental group and 368 in the control group. The basic information of the subjects is shown in Table 1.

\section{Bias assessments}

The Cochrane risk assessment tool was used for bias evaluation. The studies were randomized but only two described the correct random method (article [11] uses sealed envelopes, article [12] uses allocation lists) and noted allocation concealment. One of the RCTs was numbered according to the order of admission (high risk), whilst the other third articles referred to the admissions as "random", producing a higher likelihood of selective bias. In two of the articles, the evaluators were blind so the existence of bias and measurement bias were not excluded. All five articles had no pre-defined outcome indicators. One study had only partial outcome indicators and did not describe the specific incidence. Two of the articles lost children to follow-up, which was described by the other four articles. The risk of gender reporting was low, and there was uncertainty regarding bias from other sources. Bias assessments are shown in Figure 2-3.

\section{Meta Analysis}

\section{BDP incidence}

Six studies described the incidence of BPD, including a total of 720 children. Heterogeneity tests showed $P=0.31, P=28 \%$, suggesting no heterogeneity and so the fixed effect model was used. The analysis showed that differences in the incidence of BPD between the experimental and control groups were statistically significant [RR $=0.42,95 \% \mathrm{Cl}(0.37,0.89), P<0.001]$.

\section{BDP related mortality}

Four studies reported BPD-related mortality, including 571 children. No heterogeneity was observed between the studies ( $P=0.43 R=0 \%$ ) so a fixed effect model was employed. There were significant differences in BPD-related mortality between experimental and control groups $[\mathrm{RR}=0.54,95 \% \mathrm{Cl}(0.38$, $0.89), P<0.05]$.

\section{Secondary outcome indicators}

Intraventricular hemorrhage, infection/sepsis, the retinopathy of prematurity (ROP), patent ductus arteriosus (PDA), neonatal necrotizing enterocolitis (NEC), hyperglycemia, and hypertension were compared between experimental and control groups. No significant differences were observedbut the 
systolic blood pressure of the children in the experimental group were higher than those of the control group on the 3rd and 7th day of treatment $(P<0.05)$. The diastolic blood pressure of the children in the experimental groups were higher than that the control group on the 3rd and 5th day of treatment $(P<0.05)$.A single study [11] performed a follow-up in children aged 2 to 3 years, which showed that the body mass of the experimental and control groups [(11.6 \pm 1.4$) \mathrm{kg}$ vs $(11.4 \pm 2.6) \mathrm{Kg}]$, height $[(85.2 \pm 6.4)$ $\mathrm{cm}$ vs $(87.7 \pm 3.9) \mathrm{cm}]$, head circumference $[(46.3 \pm 1.8) \mathrm{cmvs}(47.0 \pm 3.0)$ did not significantly differ between the two groups. There were no significant differences in abnormal neurological manifestations, mental development index scores (MDI) and psychomotor development index scores (PDI) between experimental and control groups $(P>0.05)$, but only $50 \%$ of the children entered a follow-up study, so a loss of follow-up due to bias existed [17].

\section{Publication bias assessment}

Egger's tests showed no significant publication bias in the incidence of $\mathrm{BPD}[\mathrm{t}=1.04,95 \% \mathrm{Cl}(-2.12,1.86)$, $P=0.494]$ (Fig.6).

\section{Discussion}

There is no definitive definition of bronchopulmonary dysplasia (BPD). In 1979, Tooley recommended that BPD should be defined as requiring oxygen therapy 28 days after birth [18].In 1988, Shennan et al. [19] proposed that BPD should be defined as requiring clinical oxygen at 36 weeks of gestational age, which was further confirmed by Shennan and colleagues, and is now accepted in clinical practice. The latest definition of BPD refers to any oxygen dependence $\left(\mathrm{FiO}_{2}\right)>21 \%$ in newborns aged over 28 days. The pathogenesis and pathological changes of BPD are complex and caused by an array of factors. Genetic susceptibility is the basis of BPD, but other causes include persistent lung injury, abnormal repair after injury, and pulmonary dysplasia[20,21]. With the application of pulmonary surfactants, the pathological changes of new BPD are characterized by pulmonary microvascular and alveolar dysplasia.At present, there is no definitive and effective BPD treatment and its control is based on prevention. Commonly used methods include protective ventilation strategies, NO inhalation, the restriction of liquids and diuretics, caffeine, and vitamin A, active substances, glucocorticoids, and nutritional support.Since intrauterine infections such as chorioamnionitis produce inflammatory mediators during preterm birth, postnatal exposure to infections or iatrogenic factors (such as mechanical ventilation and oxygen therapy) can lead to inflammatory reactions, infections, lung dysplasia, and developmental cessation [22]. Infection and inflammation are key to BPD.Glucocorticoids have direct and indirect anti-inflammatory effects, and improve lung function through an array of mechanisms that prevent BPD occurrence, including accelerating fetal lung development, reducing pulmonary microvascular permeability, improving gas exchange, and the oxygenation index. Pulmonary edema and intrapulmonary shunts are reduced, and glucocorticoids increase the synthesis of pulmonary surfactants [23] which have received widespread attention. However, due to anincreasing number of studies highlighting how glucocorticoids increase the risk of hyperglycemia, hypertension, cardiac hypertrophy, gastrointestinal perforation, and the reduction 
of body mass, their application in premature infants remains controversial. Further research is this area is required [24].

BPD primarily occurs in premature infants, as is more obvious at a lower gestational ages. Previous studies have shown that the incidence of neonatal respiratory distress syndrome in premature infants aged 23 to 31 weeks decreases with increasing gestational age[25]. As one of the many complex multifunctional substances produced by human alveolar type II epithelial cells, pulmonary surfactants are the most important substances affecting pulmonary autonomic respiratory function[26]. Surfactants are composed of lipids and proteins and influence lung maintenance and neonatal lung surface gradient tension. To-date, four types of surfactant proteins have been isolated, amongst which proteins $B$ and $C$ interact with phospholipids to reduce the tension of the lung surface[27]. The lack of pulmonary surfactants in premature infants can directly lead to a persistent collapse of the alveoli and small airways, resulting in various dyspnea manifestations (hypoxemia and acidosis) shortly after birth, further affecting the synthesis of pulmonary surfactants[28]. With continuous improvements in perinatal medical technology, a higher number of premature infants with very low birth weights can be cured and survive BPD caused by hypoplastic lungs after air pressure injury and inflammatory factors[28]. It is thus important to prevent the occurrence of BPD in the clinic.

This study included 6 RCTs and a total of 720 preterm infants diagnosed with BPD. The results showed that pulmonary surfactants combined with budesonide intratracheal instillation reduced the incidence of BPD in preterm infants and their associated mortality, but did not increase the incidence of complications such as infection, intraventricular hemorrhage, the retinopathy of prematurity, and patent ductus arteriosus. These results are consistent with the meta-analysis of Venkataraman et al [23]. Previous studies [11] showed that pulmonary surfactants combined with intratracheal budesonide had no significant effects on growth and nervous system development in children.Nimmo et al [29] showed that pulmonary surfactants act as effective carriers of glucocorticoids in the lungs of mice.

\section{Strengths and limitations}

The limitations of this study included:the use of only 5 RCTs of which the total sample sizes were small. Four studies were from mainland China, 1 was from Taiwan, and 1 was from a multi-center research centre in Taiwan, China and Chicago, USA, so ethnic limitations existed. The ratio of pulmonary surfactants and budesonide included in the studies varied but subgroup analysis was not performed due to the small sample size. Only a single RCT was followed up, and long-term outcomes such as growth levels and nervous system development were compared. There was therefore a possibility of loss of follow-up bias and the effects of pulmonary surfactants and budesonide on the long-term development of the nervous system were unclear.

\section{Implication for research}

The efficacy and safety of PS+budesonide mixtures and intratracheal instillation with PS were not compared to other modes of administration. The scope of the study was therefore limited. In future 
studies, minimally invasive and non-invasive drug delivery methods such as nebulization should be employed to identify the optimal route of administration.

\section{Conclusions}

Pulmonary surfactants combined with the intratracheal instillation of budesonide reduce the incidence of BPD and its related mortality, and decrease the risk of short-term complications. However, its impact on longterm complications such as neurodevelopmental disorders require further studies of higher quality, that contain large sample sizes, and multi-center clinical randomization.

\section{Abbreviations}

PS:Pulmonary surfactants; BPD:Bronchopulmonary dysplasia; RCTs: Randomized controlled trials; ROP: Retinopathy of prematurity; PDA:Patent ductus arteriosus; NEC:neonatal necrotizing enterocolitis; RDS: Respiratory distress syndrome; Cl:Confidence intervals; MDI:Development index scores; RR:Relative risk ratio; PDI: Psychomotor development index scores.

\section{Declarations}

\section{Ethics approval and consent to participate}

Not applicable

\section{Consent for publication}

Not applicable

\section{Availability of data and materia}

Supporting data can be obtained from the corresponding author.

\section{Competing interests}

The authors declare that they have no competing interests.

\section{Funding}

Not applicable

\section{Authors' contributions}

The conception and design of the study was provided by Guosheng Liu; acquisition of papers, analysis and interpretation were led by Jiayong Zhuang and Boran Ye; the manuscript was first drafted by Bingbin $\mathrm{He}$; all the authors were involved in reviewing the draft. All authors have read and approved the final 
manuscript.

\section{Acknowledgements}

The authors would like to acknowledge statistical advice from staff at the University of Aberdeen.

\section{References}

1. Wei Tan,Langhuan Lei, Liping Yao,et al.Comparison of curative effects of using pulmonary surfactant at different time in treatment of premature infants with respiratory distress syndrome[J].Chinese Journal of Obstetrics \& Gynecology and Pediatrics(Electronic Edition),2017, 13(4):455-460. http://en.cnki.com.cn/Article_en/CJFDTotal-ZHFY201704015.htm

2. Feng Wang,YanMei Qiao,Liqin Chen.Effect of continuous positive airway pressure combined with pulmonary surfactant in treatment of neonatal respiratory distress syndrome[J].Maternal \& Child Health Care of China,2017, 32(13):2924-2927. http://www.wanfangdata.com.cn/details/detail.do? _type=perio\&id=zgfybj201713038.

3. Lie Huang,Xi Xu, Yongjia Ji,et al. Effect of Using Pulmonary Surfactant Mixed with Budesonide on Bronchopulmonary Dysplasia[J].Ningxia Medical Journal,2017, 39(6):511-512.

4. Mandell EW, Kratimenos $\mathrm{P}$, Abman SH,et al.Drugs for the Prevention and Treatment of Bronchopulmonary Dysplasia[J].Clin Perinatol. 2019,46(2):291-310. https://www.sciencedirect.com/science/article/pii/S0095510819300223.

5. Michael Z, Spyropoulos F, Ghanta S,et al.Bronchopulmonary Dysplasia: An Update of Current Pharmacologic Therapies and New Approaches[J].Clin Med Insights Pediatr. 2018 Dec 11;12:1179556518817322. doi: 10.1177/1179556518817322.

6. Pasha AB, Chen XQ, Zhou GP.Bronchopulmonary dysplasia: Pathogenesis and treatment[J].Exp Ther Med. 2018,16(6):4315-4321. http://www.ncbi.nlm.nih.gov/pubmed/30574005.

7. Ricci F, Catozzi C, Ravanetti F, et al.In vitro and in vivo characterization of poractant alfa supplemented with budesonide for safe and effective intratracheal administration[J].Pediatr Res. 2017,82(6):1056-1063.http://www.ncbi.nlm.nih.gov/pubmed/28723887.

8. COSTELOE K L,HENNESSY E M,HAIDER S,et al. Short term outcome after extreme preterm birth in England: Comparison of two birth cohorts in 1995 and 2006 (the EPICure studies) [J].BMJ $2012 \rrbracket$ 345: e7976囚http://www.ncbi.nlm.nih.gov/pubmed/23212881.

9. DOYLE L W, EHRENK RANZ R A,HALLIDAY H L. Early (<8days) postnatal corticosteroids for preventing chronic lung disease in preterm infants[J].Cochrane Database Syst $\mathbb{Z}$ ev,2014(5):CD001146.https://eurekamag.com/research/059/639/059639312.php.

10. SHAH S S,OHLSSON A,HALLIDAY H L,et al.Inhaled versussystemic corticosteroids for preventing chronic lung disease inventilated very low birth weight preterm neonates [J].Cochrane Database Syst Rev,2012(5) : CD002058囚http://www.ncbi.nlm.nih.gov/pubmed/22592683.

11. ]YEH T F,LIN H C,CHANG C H,et al. Early intratracheal instillation of budesonide using surfactant as a vehicle to prevent chronic lung disease in preterm infants: A pilot 
study[J].Pediatrics,2008,121(5):e1310-e1318هhttp://www.ncbi.nlm.nih.gov/pubmed/18426851

12. YEH T F,CHEN C M,WU S Y,et al.Intra-tracheal administration of budesonide/surfactant to prevent bronchopulmonary dysplasia[J].Am J Respir Crit Care Med,2016,193(1):86-95. https://www.ncbi.nlm.nih.gov/pubmed/26351971.

13. Lijing Deng,Huabao Peng,Xiaoqin Gong. Effect of budesonide combined with pulmonary surfactant on bronchopulmonary dysplasia in patients with severe respiratory distress syndrome[J].Chin $\mathrm{J}$ Neonatal.2017,32(5) : 361-364. http://www.wanfangdat a.com.cn /details /detail.do? _type=perio\&id=xsekzz201705009.

14. Zhihong Ren. Analysis on the curative effect of pulmonary surfactant combined with budesonideon prevention of bronchopulmonary dysplasia in extremely low birth weight infants[J]. Maternal and Child Health Care of China,2019,34(4):815-819. http://www.wanfangdata.com.cn/details/detail.do? _type=perio\&id=zgfybj201904030.

15. Jinzhen Su,Yulan Yang,Lin Yang,et al.The clinical study of budesonide combined with pulmonary surfactant to prevent bronchopulmonary dysplasia in premature infants[J].Int $\mathrm{J}$ Pediatr,2019,46(1):61-65.http://med.wanfangdata.com.cn/Paper/Detail?id=PeriodicalPaper_gwyxekx201901015\&dbid=WF_QK.

16. Jing Pan,Wuming Chen,Wenquan $\mathrm{Ni}$,et al. Clinical efifcacy of pulmonary surfactant combined with budesonide for preventing bronchopulmonary dysplasia in very low birth weight infants[J]. Chinese Journal of Contemporary Pediatrics,2017,19(2) : 137-

141.http://www.en.cnki.com.cn/Article_en/CJFDTotal-DDKZ201702002.htm.

17. KUO H T,LIN H C,TSAI C H,et al. A follow-up study ofpreterm infants given budesonide using surfactant as a vehicle toprevent chronic lung disease in preterm infants[J]. J Pediatr,2010,156(4) :537-541.https://www.onacademic.com/detail/journal_1000038930329310_2eae.html.

18. TOOLEY W H.Epidemiology of bronchopulmonary dysplasia[J]. J Pediatr,1979,95:851-858凶

19. SHENNAN A T,DUNN M S,OHLSSON A,et al. Abnormal pulmonary outcomes in premature infants: Prediction from oxygen requirement in the neonatal period[J]. Pediatrics,1988,82: 527532.http://www.ncbi.nlm.nih.gov/pubmed/3174313.

20. JOBE A H. What BPD in 2012 and what will BPD become? [J].Early Hum Dev,2012,88( Suppl 2) :S27S28囚https://www.onacademic.com/detail/journal_1000035084822410_25ae.html.

21. BARALDI E\FILIPPONE M $\triangle$ Chronic lung disease after premature birth[J]. N Engl J Med $₫ 2007,357$ ( 19):1946-1955.http://www.ncbi.nlm.nih.gov/pubmed/17989387.

22. SPEER C P. New insights into the pathogenesis of pulmonaryinflammation in preterm infants[J]. Biol Neonate,2001,79(3-4) : 205-209.http://www.ncbi.nlm.nih.gov/pubmed/11275652.

23. VENKATA R,AMAN R,KAMALUDDEEN M,HASAN S U, et al.Intratracheal administration of budesonidesurfactant in prevention of bronchopulmonary dysplasia in very low birth weight infants:A systematic review and Meta-analysis [J].Pediatr Pulmonol,2017,52( 7) : 968-975》 http://www.ncbi.nlm.nih.gov/pubmed/28165675. 


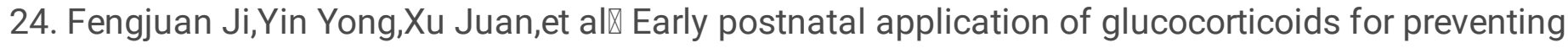
bronchopulmonary dysplasia in preterm infants: a Meta analysis[J].Chinese Journal of Contemporary Pediatrics.2017,19(6) :638-645. http://www.cnki.com.cn/Article/CJFD TotalDDKZ201706007.htm.

25. Gallini F, Arena R, Stella G, et al.Neurodevelopmental outcomes of premature infants with bronchopulmonary dysplasia[J].Acta Biomed,2014,85(1):30-34. http://www.ncbi.nlm.nih.gov/ pubmed/24957344.

26. Hascoët JM, Picaud JC, Ligi I, et al.Review shows that using surfactant a number of times or as a vehicle for budesonide may reduce the risk of bronchopulmonary dysplasia[J].Acta Paediatr, 2018,107(7):1140-1144. http://www.ncbi.nlm.nih.gov/pubmed/29193276.

27. Ghodrat M.Lung surfactants[J].Am J Health Syst Pharm, 2006,63(16):1504-1521. http://www.ncbi.nlm.nih.gov/pubmed/16896079.

28. Kazzi SN, Schürch S, McLaughlin KL, et al.Surfactant phospholipids and surface activity among preterm infants with respiratory distress syndrome who develop bronchopulmonary dysplasia[J].ActaPaediatr,2000,89(10):12181225.http://www.ncbi.nlm.nih.gov/pubmed/11083379

29. NIMMO A J,CARSTAI RS J R,PATOLE S K,et al. Intratracheal administration of glucocorticoids using surfactant as a vehicle[J].Clin Exp Pharmacol Physiol,2002,29(8):661-665区 https://eurekamag.com/research/010/876/010876399.php.

\section{Figures}


512 articles were found, 43 articles from CNKI, 35 articles from Wanfang, 112 articles from PubMed, 254 articles from EMBASE, and 68 articles from the Cochrane Library

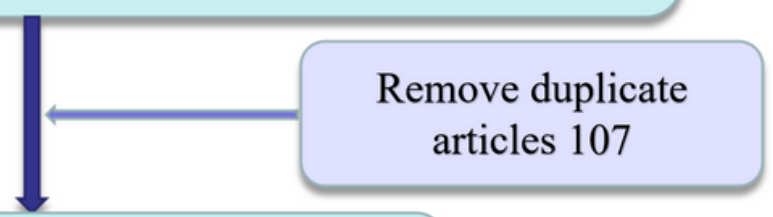

Read the literature title and abstract for preliminary screening $(n=405)$

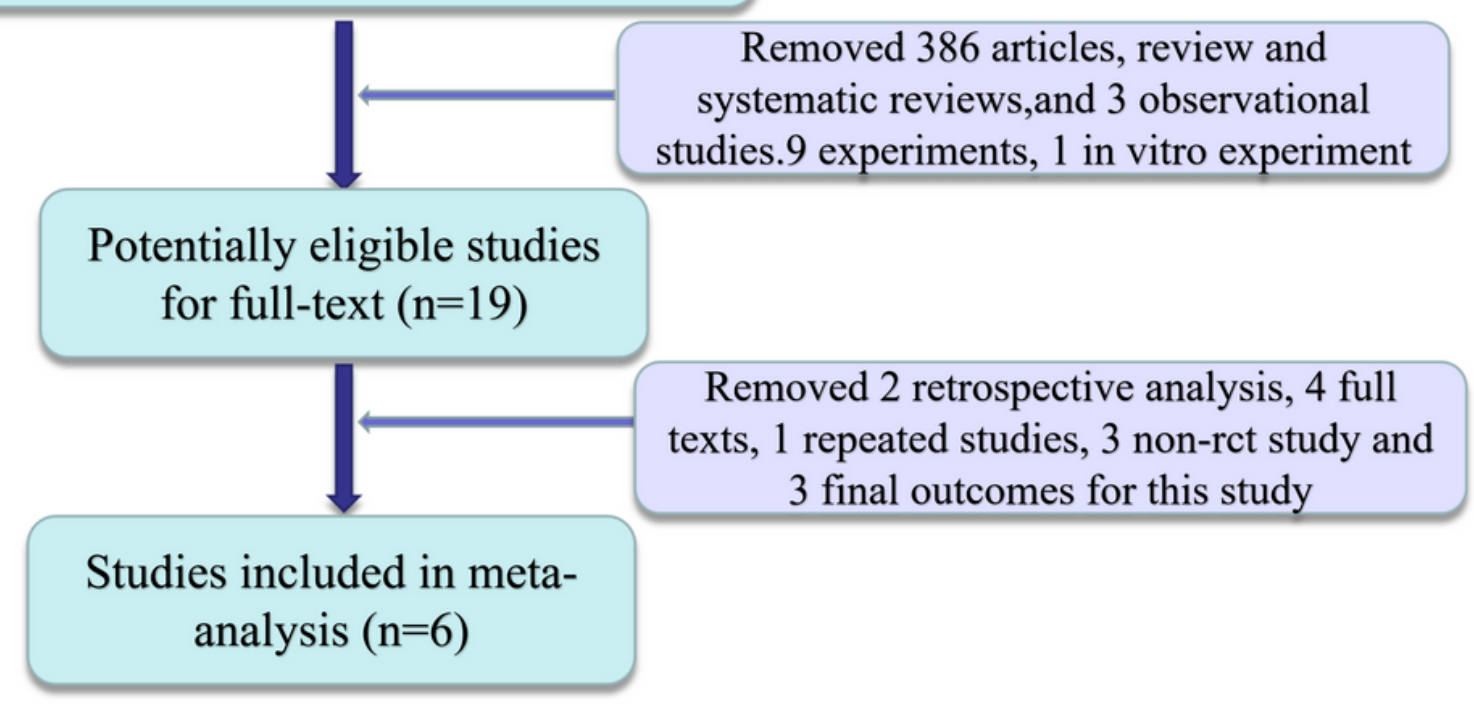

Figure 1

screening process 


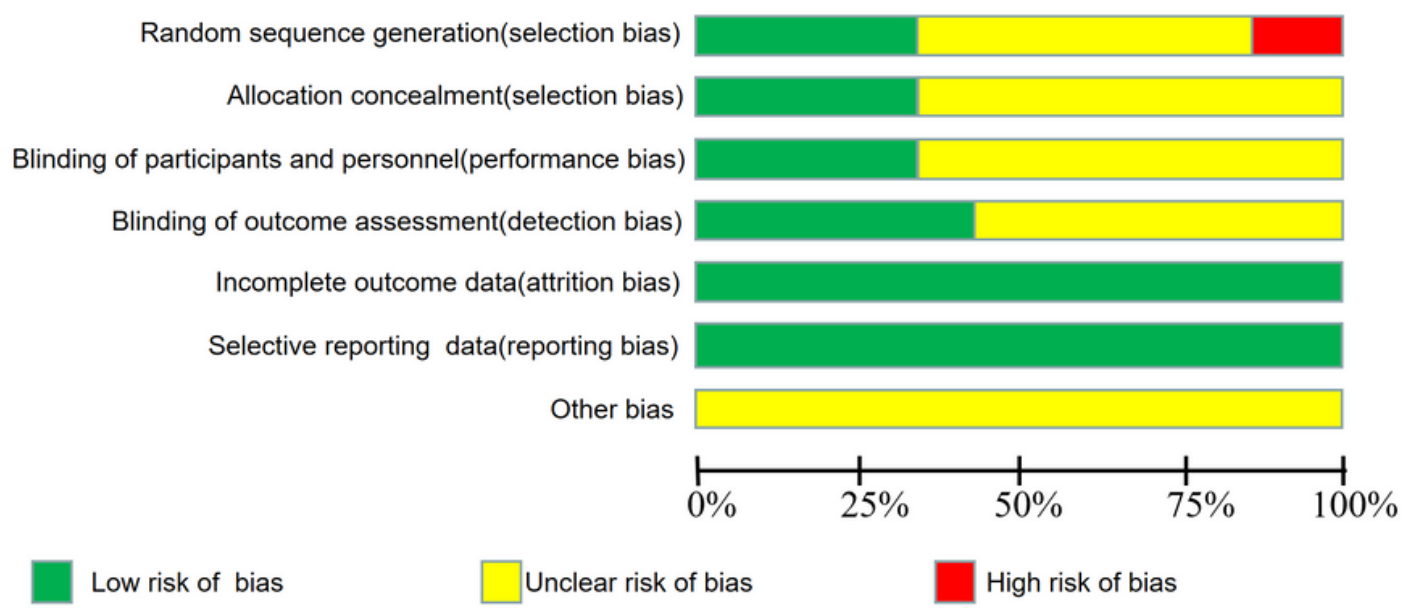

Figure 2

bias assessment 


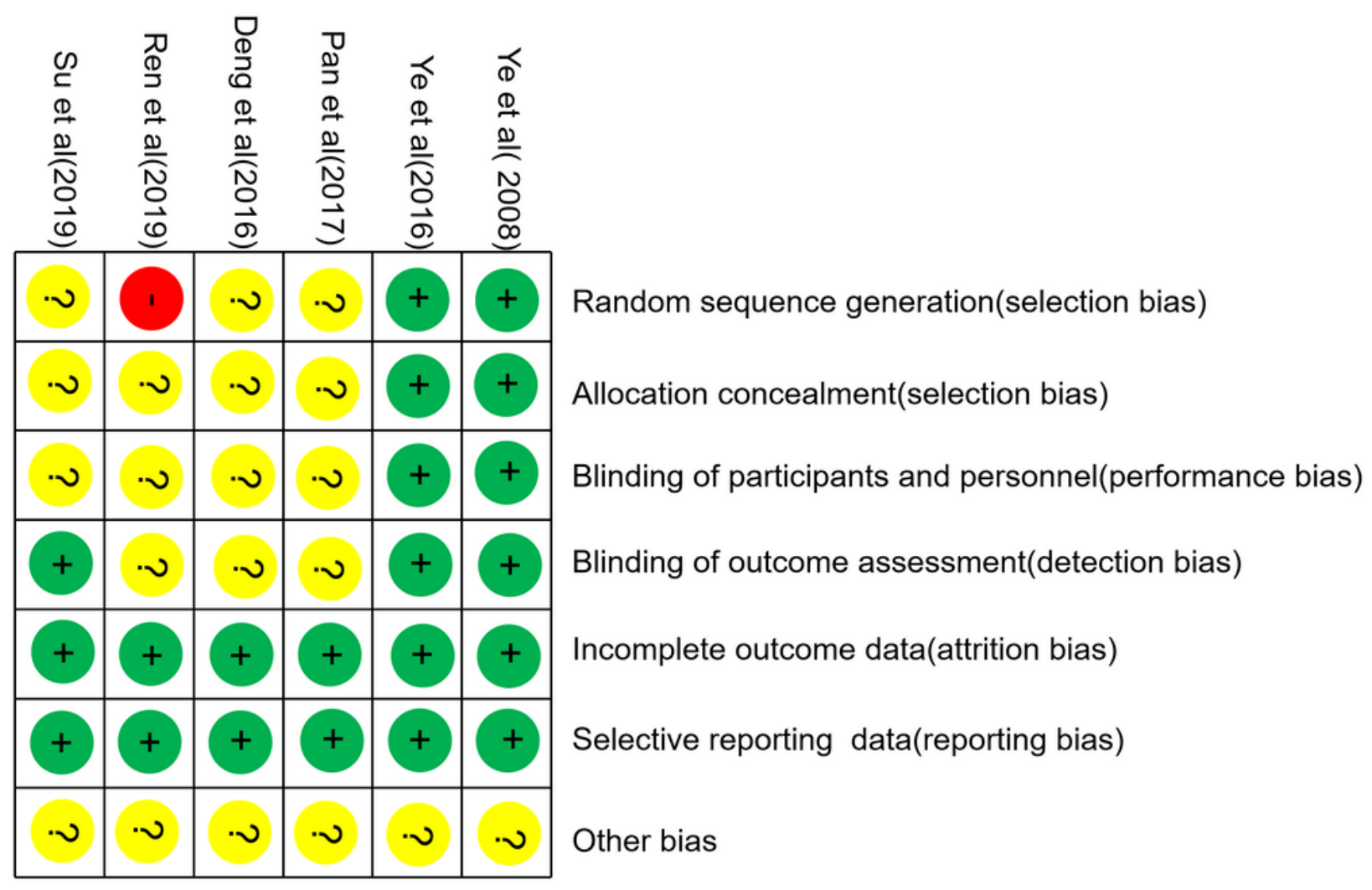

\section{Figure 3}

bias assessment 


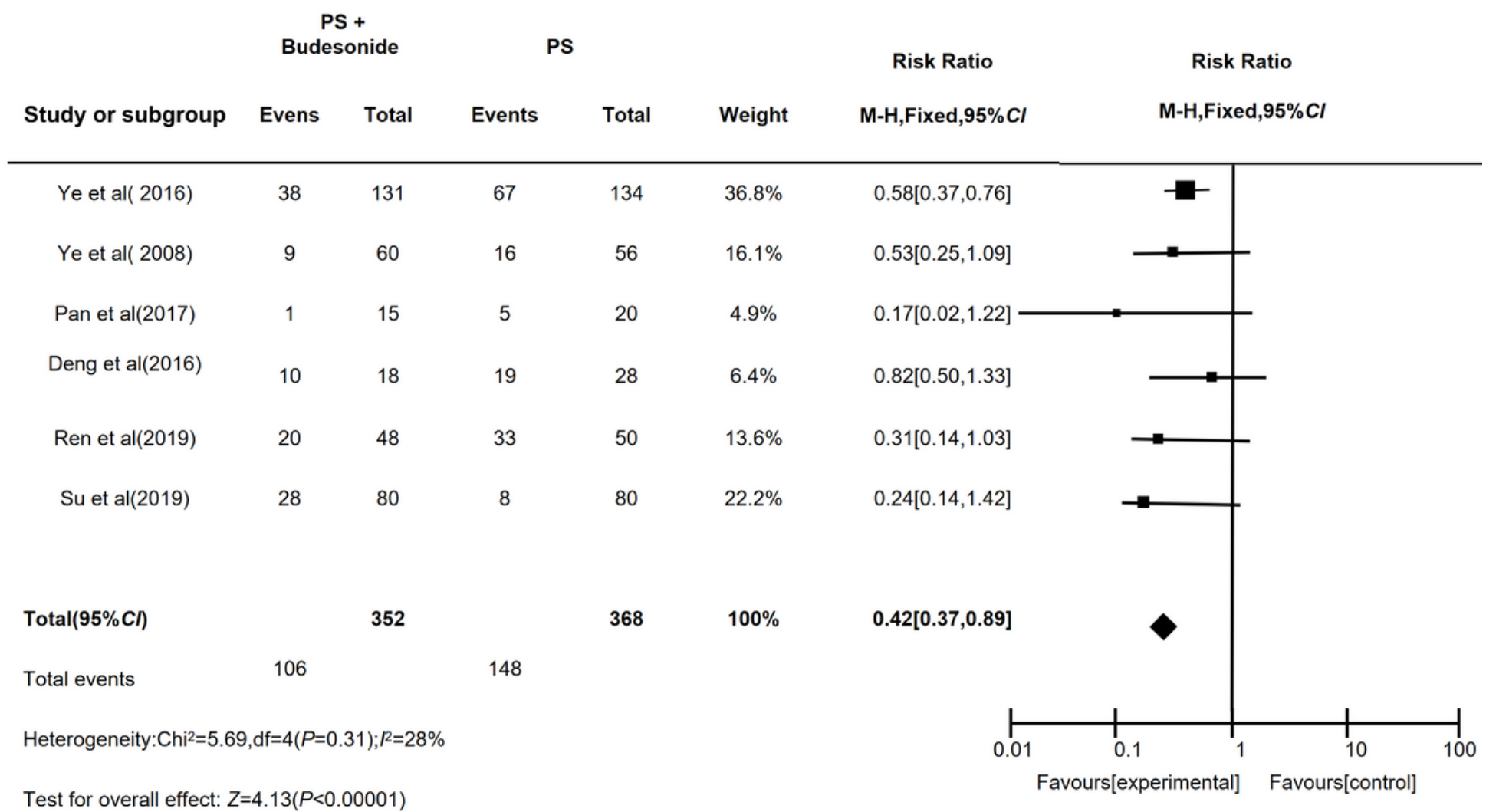

\section{Figure 4}




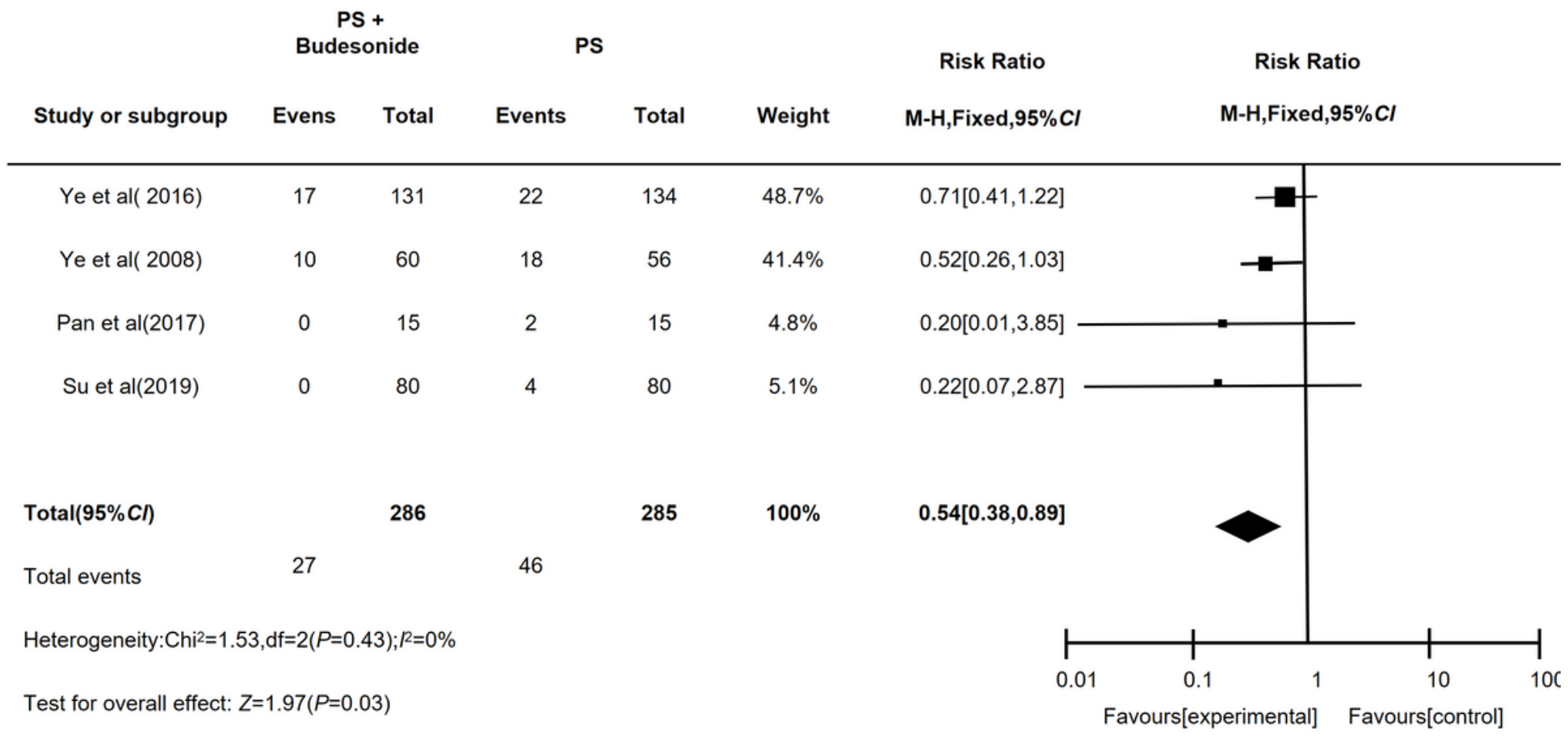

Figure 5

\begin{tabular}{|c|c|c|c|c|c|c|}
\hline Number of studi & & & & & MSE & 1.023 \\
\hline Std_Eff & Coef. & Std. Err. & $\mathbf{t}$ & $P>|t|$ & [95\% conf- & Interva] \\
\hline slope & -12411723 & .48953 & -0.27 & 0.862 & -11.482572 & 1.24571 \\
\hline bias & 1.32586 & 1.509625 & 1.04 & 0.494 & -2.119845 & 1.86432 \\
\hline
\end{tabular}

\section{Test of H0:no small-study effect}

$P=0.494$

\section{Figure 6}

publication bias assessment 


\section{Supplementary Files}

This is a list of supplementary files associated with this preprint. Click to download.

- PRISMAchecklist.pdf

- Table1.pdf

- Table2.pdf 